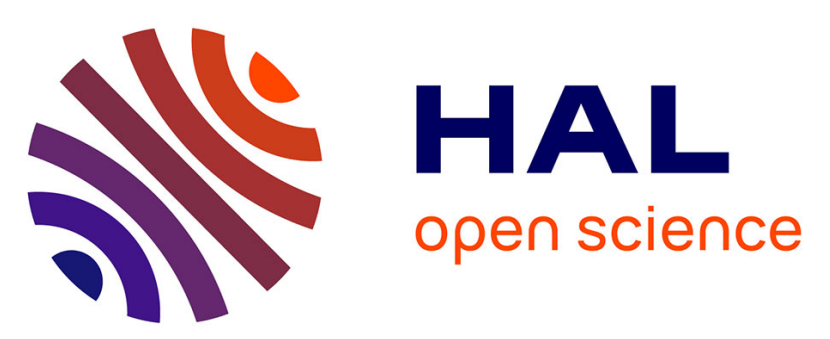

\title{
Effects of slip on the Von Kármán swirling flow and heat transfer in a porous medium
}

Bikash Sahoo, Sébastien Poncet, Fotini Labropulu

\section{To cite this version:}

Bikash Sahoo, Sébastien Poncet, Fotini Labropulu. Effects of slip on the Von Kármán swirling flow and heat transfer in a porous medium. Transactions of the Canadian Society for Mechanical Engineering, 2015, 39 (2), pp.357-366. 10.1139/tcsme-2015-0025 . hal-01300117

\section{HAL Id: hal-01300117 \\ https://hal.science/hal-01300117}

Submitted on 23 Oct 2020

HAL is a multi-disciplinary open access archive for the deposit and dissemination of scientific research documents, whether they are published or not. The documents may come from teaching and research institutions in France or abroad, or from public or private research centers.
L'archive ouverte pluridisciplinaire HAL, est destinée au dépôt et à la diffusion de documents scientifiques de niveau recherche, publiés ou non, émanant des établissements d'enseignement et de recherche français ou étrangers, des laboratoires publics ou privés. 


\title{
EFFECTS OF SLIP ON THE VON KÁRMÁN SWIRLING FLOW AND HEAT TRANSFER IN A POROUS MEDIUM
}

\author{
Bikash Sahoo ${ }^{1}$, Sébastien Poncet ${ }^{2,3}$ and Fotini Labropulu ${ }^{4}$ \\ ${ }^{1}$ Department of Mathematics, National Institute of Technology Rourkela, Rourkela, India \\ ${ }^{2}$ Faculté de Génie, Université de Sherbrooke, Sherbrooke, Canada \\ ${ }^{3}$ Aix-Marseille Université, CNRS, École Centrale, Laboratoire M2P2 UMR 7340, Marseille, France \\ ${ }^{4}$ Department of Mathematics, Luther College, University of Regina, Regina, Canada \\ E-mail: bikashsahoo@nitrkl.ac.in; Sebastien.Poncet@USherbrooke.ca; fotini.labropulu@uregina.ca
}

\begin{abstract}
Numerical solutions are obtained for the fully coupled and highly nonlinear system of differential equations, arising due to the steady Kármán flow and heat transfer of a viscous fluid in a porous medium. The conventional no-slip boundary conditions are replaced by partial slip boundary conditions owing to the roughness of the disk surface. Combined effects of the slip $\lambda$ and porosity $\gamma$ parameters on the momentum and thermal boundary layers are studied in detail. Both parameters produce the same effects on the mean velocity profiles, such that all velocity components are reduced by increasing either $\lambda$ or $\gamma$. The temperature slip factor $\beta$ has a dominating influence on the temperature profiles by decreasing the fluid temperature in the whole domain. The porosity parameter strongly decreases the heat transfer coefficient at the wall for low values of $\beta$ and tends to an asymptotical limit around 0.1 for $\beta \simeq 10$. The porosity parameter $\gamma$ increases the moment coefficient at the disk surface, which is found to monotonically decrease with $\lambda$.
\end{abstract}

Keywords: Kármán flow; porous medium; partial slip.

\section{EFFETS DES CONDITIONS D’ADHÉRENCE SUR L'ÉCOULEMENT DE VON KÁRMÁN CHAUFFÉ DANS UN MILIEU POREUX}

\section{RÉSUMÉ}

Des solutions numériques ont été obtenues pour l'écoulement stationnaire de von Kármán anisotherme dans un milieu poreux. Les conditions classiques de non-glissement à la surface du disque sont remplacées par des conditions de glissement partiel permettant de simuler un disque rugueux. Les effets combinés des paramètres de glissement $\lambda$ et de porosité $\gamma$ sur les couches limites hydrodynamique et thermique sont étudiés en détails. Ces deux paramètres ont les mêmes effets sur les profils de vitesse, de telle sorte que toutes les composantes de vitesse sont réduites lorsque $\lambda$ ou $\gamma$ augmente. Le facteur de glissement pour la température $\beta$ a une influence primordiale sur les profils de température en diminuant la température du fluide dans tout le domaine. La porosité induit une forte chute du coefficient de transfert de chaleur à la paroi pour des faibles valeurs de $\beta$ et tend vers une valeur asymptotique autour de 0.1 pour $\beta \simeq 10$. La porosité $\gamma$ augmente le coefficient de moment à la surface du disque, alors que celui-ci décroît monotoniquement avec $\lambda$.

Mots-clés : écoulement de Kármán; milieu poreux; conditions d'adhérence partielle. 


\section{NOMENCLATURE}

\begin{tabular}{|ll|}
\hline$C_{m}$ & dimensionless moment coefficient $(-)$ \\
$c_{p}$ & heat capacity of the fluid at constant pressure $(\mathrm{J} / \mathrm{kg} / \mathrm{K})$ \\
$F, G, H$ & normalized radial, tangential and axial velocity components $(-)$ \\
$K$ & Darcy permeability $\left(\mathrm{m}^{2}\right)$ \\
$N u$ & Nusselt number $(-)$ \\
$p, P$ & pressure $(\mathrm{Pa})$ and normalized pressure $(-)$ \\
$P r$ & Prandtl number $(-)$ \\
$q$ & heat flux supplied to the disk $\left(\mathrm{W} / \mathrm{m}^{2}\right)$ \\
$r, \phi, z$ & cylindrical coordinates $(\mathrm{m})$ \\
$R e$ & rotational Reynolds number $(-)$ \\
$T$ & temperature $(\mathrm{K})$ \\
$u, v, w$ & radial, tangential and axial velocity components $(\mathrm{m} / \mathrm{s})$ \\
$\beta$ & normalized temperature slip factor $(-)$ \\
$\beta_{1}$ & proportionality constant $(-)$ \\
$\varepsilon$ & porosity $(-)$ \\
$\gamma$ & normalized porosity parameter $(-)$ \\
$\kappa$ & thermal conductivity of the fluid $(\mathrm{W} /(\mathrm{m} \cdot \mathrm{K}))$ \\
$\lambda, \eta$ & normalized velocity slip parameters $(-)$ \\
$\mu, v$ & dynamic $($ Pa.s $)$ and kinematic $\left(\mathrm{m}^{2} / \mathrm{s}\right)$ fluid viscosities \\
$\Omega$ & rotation rate of the disk (rad $/ \mathrm{s})$ \\
$\rho$ & voluminal mass of the fluid $\left(\mathrm{kg} / \mathrm{m}^{3}\right)$ \\
$\zeta$ & normalized distance from the disk $(-)$ \\
$\theta$ & normalized temperature $(-)$ \\
$w$ & denotes a quantity evaluated at the wall \\
$\infty$ & denotes a quantity evaluated at infinity \\
, & denotes a derived quantity according to the axial direction \\
\hline
\end{tabular}

\section{INTRODUCTION}

Swirling flows are one of the classical problems in fluid mechanics. They have indeed many interesting features and industrial applications: rotating machineries, nuclear reactor or computer storage devices among other things. Porous and rough rotating disks are also used in the chemical industry as electrodes to catalyse or promote electrochemical reactions [1] and in process engineering for ultra-fine filtration [2].

The steady flow of an incompressible viscous liquid due to an infinite rotating disk was first discussed by von Kármán in 1921 [3]. Sharp gradients in the centrifugal force across the boundary layer can generate high velocities along the bounding surfaces resulting in a secondary flow. Little attention has been paid to its strong influence on the primary rotating flow outside the boundary layer. Many configurations with noslip boundary conditions have been considered in the past by many researchers including various physical effects. One can refer to the works by Rott and Lewellen [4], Owen and Rogers [5], Zandbergen and Dijkstra [6] and all references herein for a state-of-art about von Kármán swirling flows.

However, swirling flows subject to partial slip boundary conditions are more practical in nature and mathematically challenging. Effects of slip and suction on swirling flows have been thoroughly investigated by Miklavčič and Wang [7] and Turkyilmazoglu and Senel [8]. Joseph [9] has reported that the suction parameter may be identified to the permeability parameter, which depends itself on the Darcy's law and accounts for the drag exerted by the porous medium. Recently, Attia [10] solved the system of differential equations for the steady flow over a rotating disk in porous medium with heat transfer. It has been extended in a later paper to the cases where the porosity parameter tends either to zero or infinity [11]. Literature survey reveals that there is not a single report on the combined effects of slip and porosity on the swirling flow. 
This paper is an endeavor to fill this gap by providing reference data. In this note, one will mainly focus on the combined effects of velocity slip and porosity on the von Kármán swirling flow and heat transfer of a viscous fluid for a rough rotating disk. The temperature jump arising due to the velocity slip has also been considered.

\section{FORMULATION OF THE PROBLEM}

One considers a viscous incompressible fluid occupying the space $z>0$ over an infinite porous disk, which coincides with $z=0$. The steady motion is due to the rotation of the disk at a constant rotation rate $\Omega$ through a porous medium, where the Darcy model is assumed $[10,12]$. In view of the rotational symmetry $(\partial / \partial \phi \equiv 0)$, the flow is described, in the cylindrical polar coordinates $(r, \phi, z)$, by the following set of equations:

$$
\begin{aligned}
\frac{\partial u}{\partial r}+\frac{u}{r}+\frac{\partial w}{\partial z} & =0 \\
\rho\left(u \frac{\partial u}{\partial r}+w \frac{\partial u}{\partial z}-\frac{v^{2}}{r}\right)+\frac{\partial p}{\partial r} & =\mu\left(\frac{\partial^{2} u}{\partial r^{2}}+\frac{1}{r} \frac{\partial u}{\partial r}-\frac{u}{r^{2}}+\frac{\partial^{2} u}{\partial z^{2}}\right)-\frac{\mu}{K \varepsilon} u \\
\rho\left(u \frac{\partial v}{\partial r}+w \frac{\partial v}{\partial z}+\frac{u v}{r}\right) & =\mu\left(\frac{\partial^{2} v}{\partial r^{2}}+\frac{1}{r} \frac{\partial v}{\partial r}-\frac{v}{r^{2}}+\frac{\partial^{2} v}{\partial z^{2}}\right)-\frac{\mu}{K \varepsilon} v \\
\rho\left(u \frac{\partial w}{\partial r}+w \frac{\partial w}{\partial z}\right)+\frac{\partial p}{\partial z} & =\mu\left(\frac{\partial^{2} w}{\partial r^{2}}+\frac{1}{r} \frac{\partial w}{\partial r}+\frac{\partial^{2} w}{\partial z^{2}}\right)-\frac{\mu}{K \varepsilon} w
\end{aligned}
$$

where $\mathbf{V}=(u, v, w)$ is the fluid velocity vector, $p$ the pressure, $\rho$ the fluid density, $\mu$ the fluid dynamic viscosity, $K$ the Darcy permeability and $\varepsilon$ the porosity. Using the von Kármán transformations [3], the following non-dimensional functions of $\zeta$ are introduced:

$$
\begin{aligned}
& \zeta=\frac{z}{\sqrt{\frac{v}{\Omega}}}, \\
& F(\zeta)=\frac{u(z)}{\Omega r}, \quad G(\zeta)=\frac{v(z)}{\Omega r}, \quad H(\zeta)=\frac{w(z)}{\sqrt{\Omega v}}, \quad P=\frac{p-p_{\infty}}{-\rho \nu \Omega}
\end{aligned}
$$

where $F, G, H$ and $P$ are the normalized velocity components and pressure respectively, $\zeta$ the nondimensional distance measured along the rotation axis and $v$ the fluid kinematic viscosity. Following Attia [10] and Rashidi et al. [12], the homogenized equations of continuity and motion, in a spatially periodic porous medium in the $\phi$ direction, take the form:

$$
\begin{aligned}
& \frac{d H}{d \zeta}+2 F=0, \\
& \frac{d^{2} F}{d \zeta^{2}}-H \frac{d F}{d \zeta}-F^{2}+G^{2}-\gamma F=0 \\
& \frac{d^{2} G}{d \zeta^{2}}-H \frac{d G}{d \zeta}-2 F G-\gamma G=0 \\
& \frac{d^{2} H}{d \zeta^{2}}-H \frac{d H}{d \zeta}+\frac{d P}{d \zeta}-\gamma H=0
\end{aligned}
$$

This set of equations is valid under the assumption that the Reynolds number remains small enough $R e \ll 1$ to neglect the Forchheimer correction factor $F=O(R e)$ [13]. 


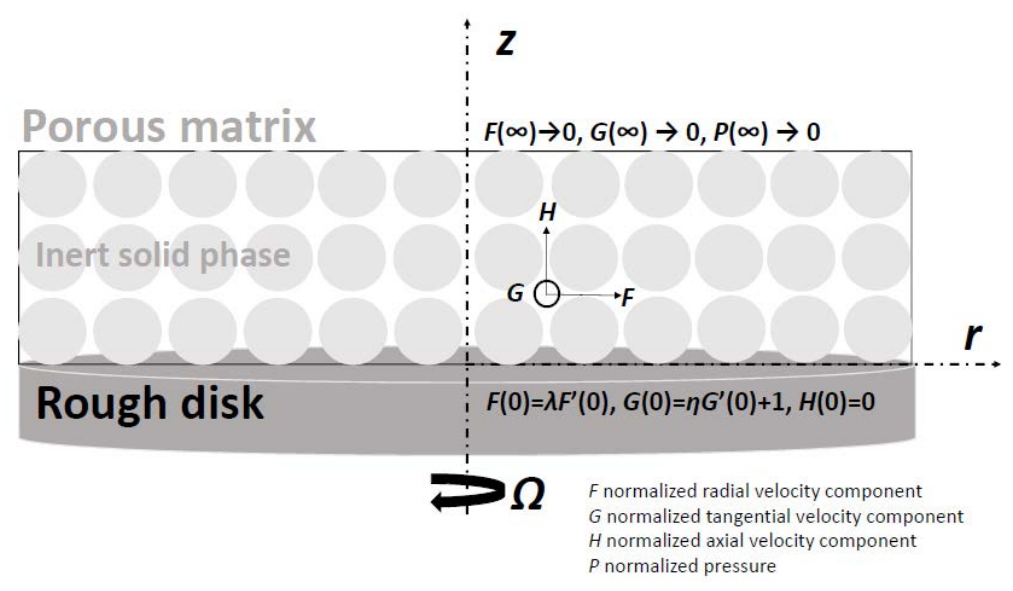

Fig. 1. Schematic representation of the Von Kármán flow in a porous medium with relevant boundary conditions.

The partial slip boundary conditions $[7,8,14]$ are introduced as:

$$
\left.u\right|_{z=0}=\left.\lambda_{1} \overline{\tau_{r z}}\right|_{z=0},\left.\quad v\right|_{z=0}=\left.\lambda_{2} \overline{\tau_{\phi z}}\right|_{z=0}
$$

where $\overline{\tau_{r z}}$ and $\overline{\tau_{\phi z}}$ are the wall shear stresses, and $\lambda_{1}$ and $\lambda_{2}$ two coefficients. The boundary conditions for the velocity problem in their dimensionless form are given by

$$
\begin{gathered}
F(\zeta=0)=\lambda F^{\prime}(\zeta=0), \quad G(\zeta=0)=1+\eta G^{\prime}(\zeta=0), \quad H(\zeta=0)=0, \\
F(\zeta \rightarrow \infty) \rightarrow 0, \quad G(\zeta \rightarrow \infty) \rightarrow 0, \quad P(\zeta \rightarrow \infty) \rightarrow 0 .
\end{gathered}
$$

where $\gamma=\frac{v}{K \Omega \varepsilon}$ is the porosity parameter. A sketch of the flow configuration with the relevant boundary conditions is displayed in Figure 1. In Eq. (11), $\lambda$ and $\eta$ are the non-dimensional slip parameters defined as

$$
\lambda=\lambda_{1} \sqrt{\frac{\Omega}{v}} \mu, \quad \eta=\lambda_{2} \sqrt{\frac{\Omega}{v}} \mu
$$

For sake of clarity, one will set $\lambda=\eta$ in all calculations.

Due to the temperature difference between the disk surface and the ambient fluid, heat transfer takes place. One will assume, as a first step, that the solid matrix is thermally inert. Thus, the energy equation without viscous dissipation takes the form:

$$
\rho c_{p}\left(u \frac{\partial T}{\partial r}+w \frac{\partial T}{\partial z}\right)-\kappa\left(\frac{\partial^{2} T}{\partial z^{2}}+\frac{\partial^{2} T}{\partial r^{2}}+\frac{1}{r} \frac{\partial T}{\partial r}\right)=0,
$$

where $c_{p}$ is the specific heat at constant pressure and $\kappa$ is the thermal conductivity of the fluid. Introducing the non-dimensional temperature $\theta=\frac{T-T_{\infty}}{T_{w}-T_{\infty}}$ and using the aforementioned von Kármán transformations, Eq. (13) becomes

$$
H \frac{d \theta}{d \zeta}=\frac{1}{\operatorname{Pr}} \frac{d^{2} \theta}{d \zeta^{2}}
$$

where $T_{w}$ is the wall temperature, $T_{\infty}$ is the temperature of the ambient fluid at a large distance from the disk, $\operatorname{Pr}=\frac{c_{p} \mu}{\kappa}$ is the Prandtl number. A temperature slip condition similar to the Navier's condition is introduced $[8,15]$ :

$$
T-T_{w}=\left.\beta_{1} \partial \frac{\partial z}{z}\right|_{z=0}
$$


where $\beta_{1}$ is a proportionality constant. The boundary conditions in terms of the non-dimensional parameter $\theta$ are expressed as

$$
\begin{aligned}
& \theta(\zeta=0)=1+\beta \theta^{\prime}(\zeta=0) \\
& \theta(\zeta \rightarrow \infty) \rightarrow 0
\end{aligned}
$$

where $\beta=\beta_{1} \sqrt{\Omega / v}$ is the thermal slip factor. The heat transfer from the disk surface to the fluid is computed by the application of the Fourier's law, $q=-\kappa\left(\frac{\partial T}{\partial z}\right)_{w}$. Introducing the transformed variables, the expression for $q$ becomes

$$
q=-\kappa\left(T_{w}-T_{\infty}\right) \sqrt{\frac{\Omega}{v}} \frac{d \theta(0)}{d \zeta}
$$

By rephrasing the heat transfer results in terms of the Nusselt number defined as

$$
N u=\frac{q \sqrt{\frac{v}{\Omega}}}{\kappa\left(T_{w}-T_{\infty}\right)},
$$

one obtains

$$
N u=-\left.\frac{d \theta}{d \zeta}\right|_{\zeta=0}
$$

\section{NUMERICAL SOLUTION}

Here one has adopted the direct multiple shooting method to solve the system of nonlinear differential equations (6)-(9) and (14) subject to the slip boundary conditions (11) and (16) in the infinite domain $0 \leq$ $\zeta<\infty$. A finite domain $0 \leq \zeta \leq \zeta_{\infty}$ can be used instead with $\zeta_{\infty}$ chosen large enough to ensure the correct asymptotic conditions at a finite distance from the disk. Computations have been carried out for $\zeta_{\infty}=14$ to be compared with the value $\zeta_{\infty}=12$, used by Attia [10]. The whole domain of integration $\left[0, \zeta_{\infty}\right]$ has been divided into subintervals by introducing additional grid points $\zeta_{0}=0<\zeta_{1}<\zeta_{2}<\ldots<\zeta_{N}=\zeta_{\infty}$. The aforementioned system of equations can be written as a system of seven first-order equations. One sets:

$$
\begin{gathered}
y_{1}=F, \quad y_{2}=G, \quad y_{3}=H, \quad y_{4}=F^{\prime}, \\
y_{5}=G^{\prime}, \quad y_{6}=P, \quad y_{7}=\theta, \quad y_{8}=\theta^{\prime}
\end{gathered}
$$

Equations (6)-(9) and (14) can be rewritten as

$$
\begin{aligned}
& \frac{d y_{1}}{d \zeta}=y_{4} ; \quad y_{1}(0)=\lambda s_{1} \\
& \frac{d y_{2}}{d \zeta}=y_{5} ; \quad y_{2}(0)=1+\eta s_{2} \\
& \frac{d y_{3}}{d \zeta}=-2 y_{1} ; \quad y_{3}(0)=0 \\
& \frac{d y_{4}}{d \zeta}=y_{3} y_{4}+y_{1}^{2}-y_{2}^{2}+\gamma y_{1} ; \quad y_{4}(0)=s_{1}
\end{aligned}
$$


Table 1. Variations of $G^{\prime}(0)$ with $\lambda$ and $\eta$ for $\gamma=0$.

\begin{tabular}{cccc}
\hline$\lambda$ & $\eta$ & \multicolumn{2}{c}{$G^{\prime}(0)$} \\
\hline & & Miklavčič and Wang [7] & Current result \\
\hline 0.0 & 0.0 & -0.61592201 & -0.61592118 \\
0.0 & 0.1 & -0.56451092 & -0.56451083 \\
0.1 & 0.1 & -0.60583524 & -0.60583521 \\
0.0 & 0.2 & -0.52202696 & -0.52202692 \\
0.1 & 0.2 & -0.55643671 & -0.55643669 \\
2.0 & 10.0 & -0.07948551 & -0.07948548 \\
5.0 & 10.0 & -0.08054447 & -0.08054443 \\
10.0 & 10.0 & -0.08103009 & -0.08103002 \\
\hline
\end{tabular}

$$
\begin{aligned}
& \frac{d y_{5}}{d \zeta}=y_{3} y_{5}+2 y_{1} y_{2}+\gamma y_{2} ; \quad y_{5}(0)=s_{2} \\
& \frac{d y_{6}}{d \zeta}=2 y_{4}-2 y_{1} y_{3}+\gamma y_{3} ; \quad y_{6}(0)=s_{3} \\
& \frac{d y_{7}}{d \zeta}=y_{8} ; \quad y_{7}(0)=1+\beta s_{4} \\
& \frac{d y_{8}}{d \zeta}=\operatorname{Pr}_{3} y_{8} ; \quad y_{8}(0)=s_{4}
\end{aligned}
$$

The initial guesses for $y_{i}$ at each nodal points are wisely chosen. The solutions obtained in each interval are pieced together to form continuous trajectories of the velocity and temperature profiles. Utmost care has been taken while refining values of the missing initial guesses $s_{1}, s_{2}, s_{3}$ and $s_{4}$ by the Broyden's method [16].

\section{RESULTS}

To validate the numerical method, the computed values of the velocity gradient $G^{\prime}(0)$ are compared for $\gamma=0$ with the numerical solutions reported by Miklavčič and Wang [7]. As shown in Table 1, a very good agreement has been obtained with a maximum difference less than $10^{-6}$ on the axial gradient of the dimensionless tangential velocity at the disk surface. The present solver can be now used extensively to investigate the combined effects of the porosity and slip coefficients on the velocity components and the temperature distribution.

Figures 2(a) and (b) elucidate the effects of the velocity slip and porosity parameters on the radial velocity component $F$. It decreases with increase values of either $\lambda$ or $\gamma$. However, the influence of porosity on $F$ is prominent near the disk surface. The axial distributions of the azimuthal velocity component $G$ are shown for various values of $\lambda$ and $\gamma$ in Figs. 3(a) and (b) respectively. It decays exponentially in a monotonic fashion as one moves away from the wall. The tangential velocity component is strongly reduced also for increasing values of either $\lambda$ or $\gamma$. Increasing the porosity parameter decreases also the thickness of the boundary layer developed along the disk. Figure 4(b) depicts that porosity has a significant effect on the axial velocity component $-H$, which decreases with an increase in $\gamma$ throughout the integration domain. The same effect is also induced by increasing the slip parameter $\lambda$ (Fig. 4a).

To investigate the heat transfer process, the Prandtl number has been fixed to $\operatorname{Pr}=5$ to simulate a water flow. Figures 5a-5c show the axial distributions of the non-dimensional temperature $\theta$ with $\lambda, \gamma$ and $\beta$ when other flow parameters are kept constant. Clearly, temperature increases with an increase in velocity slip and porosity. From Fig. 5(b), again it is evident that porosity has a tremendous effect on the thermal boundary layer. The absence of fluid at a near-ambient temperature close to the wall increases the heat transfer. Interestingly, the temperature jump $\beta$ has an opposite effect on $\theta$, as shown in Fig. 5(c). 


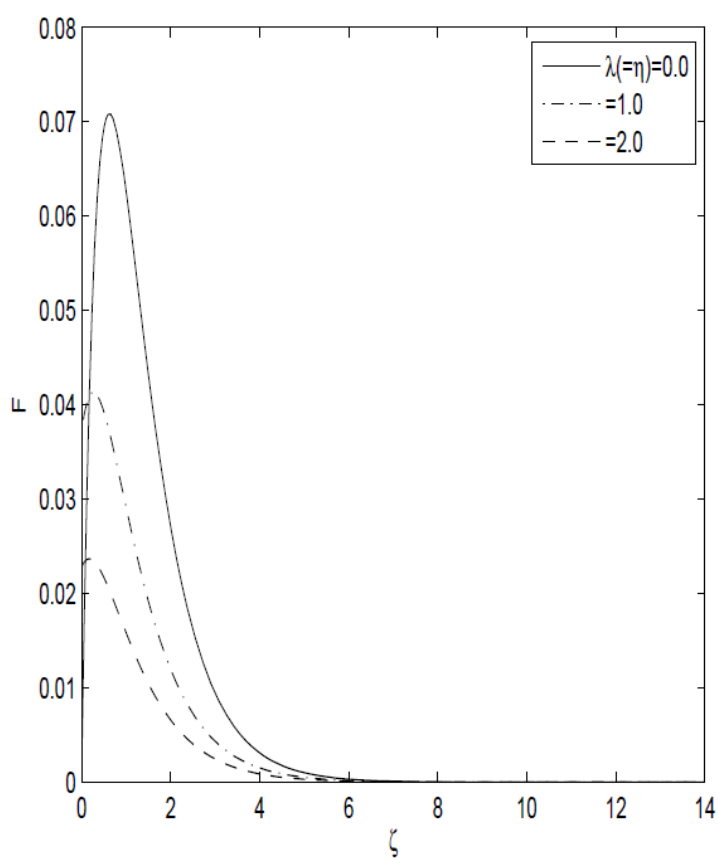

(a)

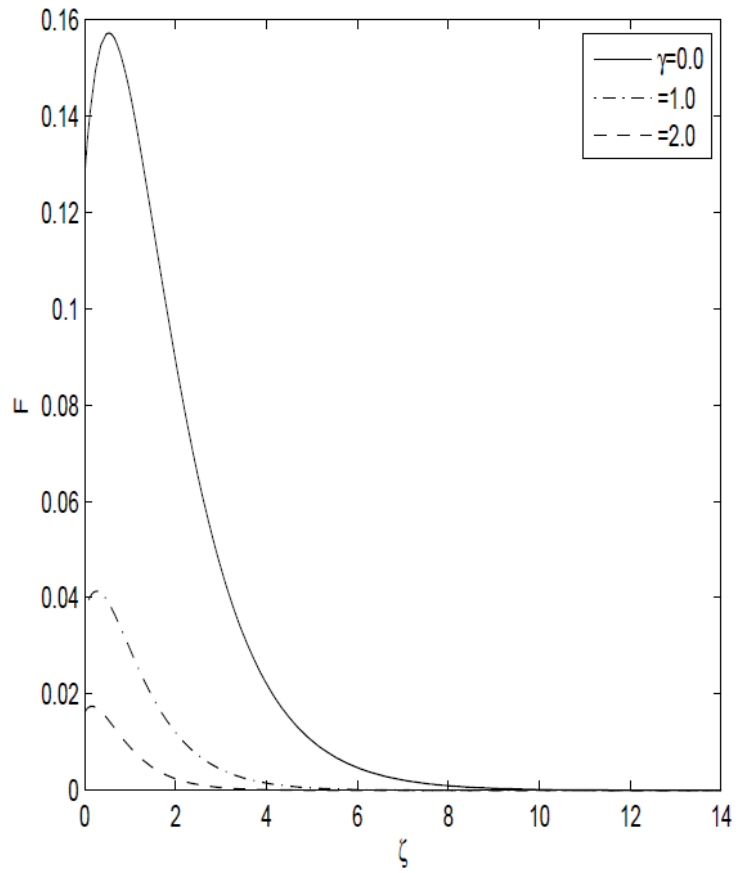

(b)

Fig. 2. Axial distributions of the normalized radial velocity component $F$ with (a) the velocity slip parameter $\lambda(=\eta)$ for $\gamma=1$ and (b) the porosity parameter $\gamma$ for $\lambda=\eta=1$.

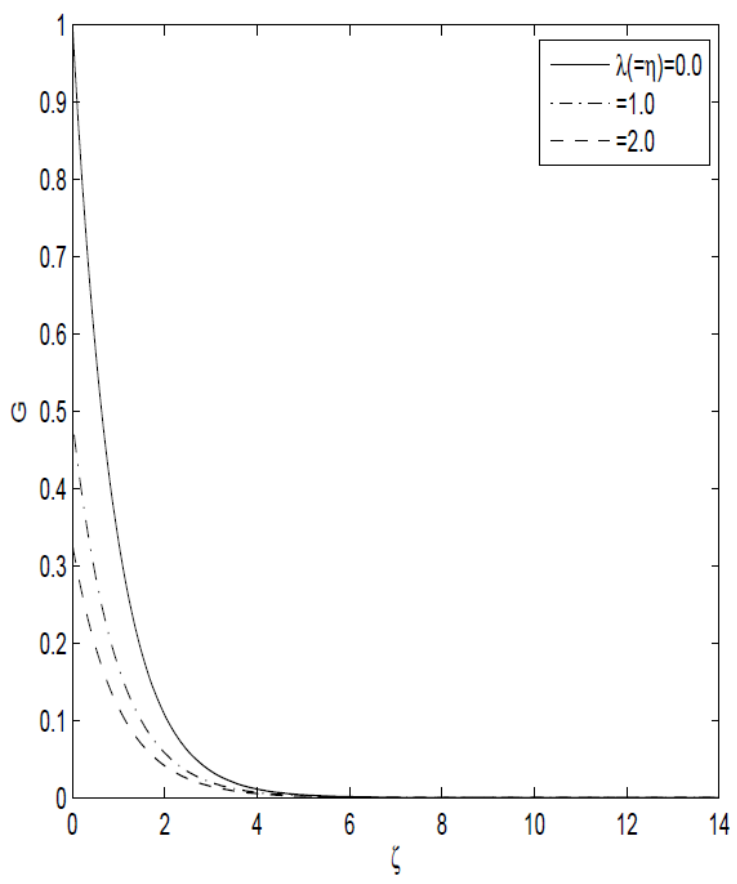

(a)

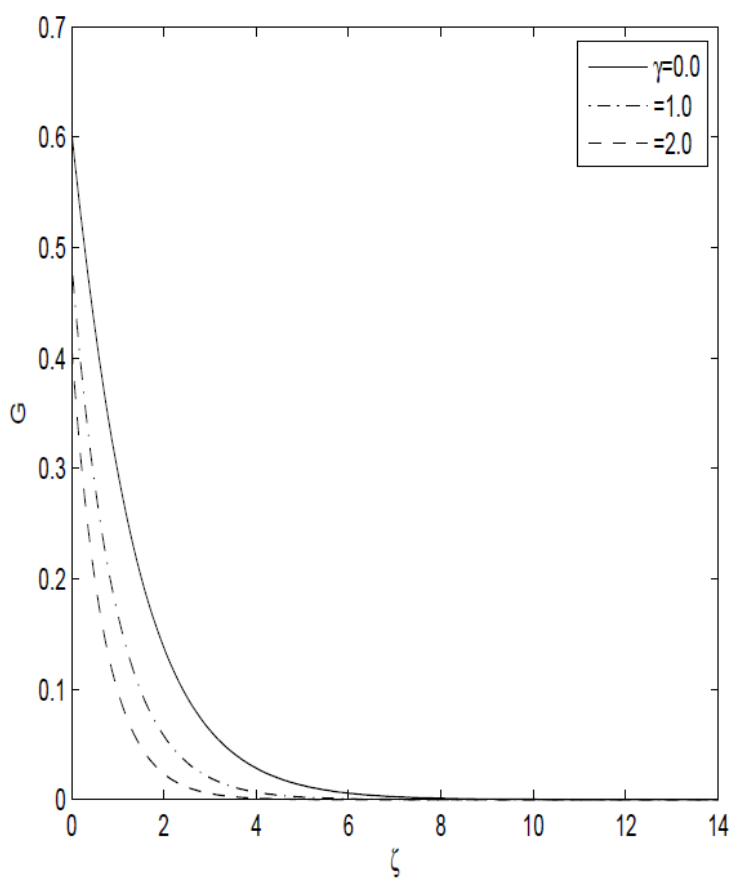

(b)

Fig. 3. Axial distributions of the normalized tangential velocity component $G$ with (a) the velocity slip parameter $\lambda(=\eta)$ for $\gamma=1$ and (b) the porosity parameter $\gamma$ for $\lambda=\eta=1$. 


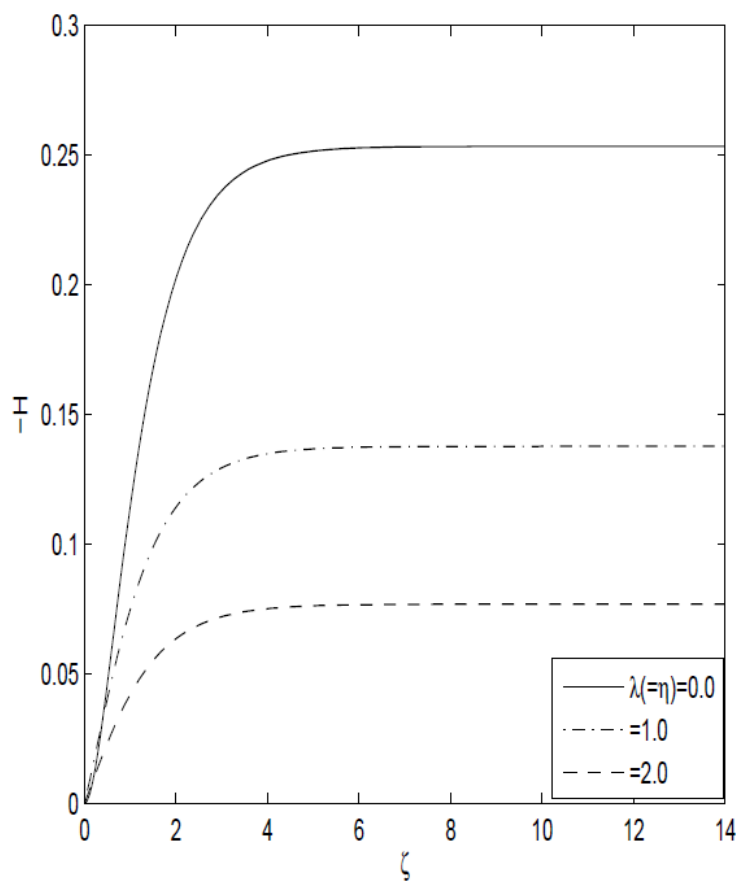

(a)

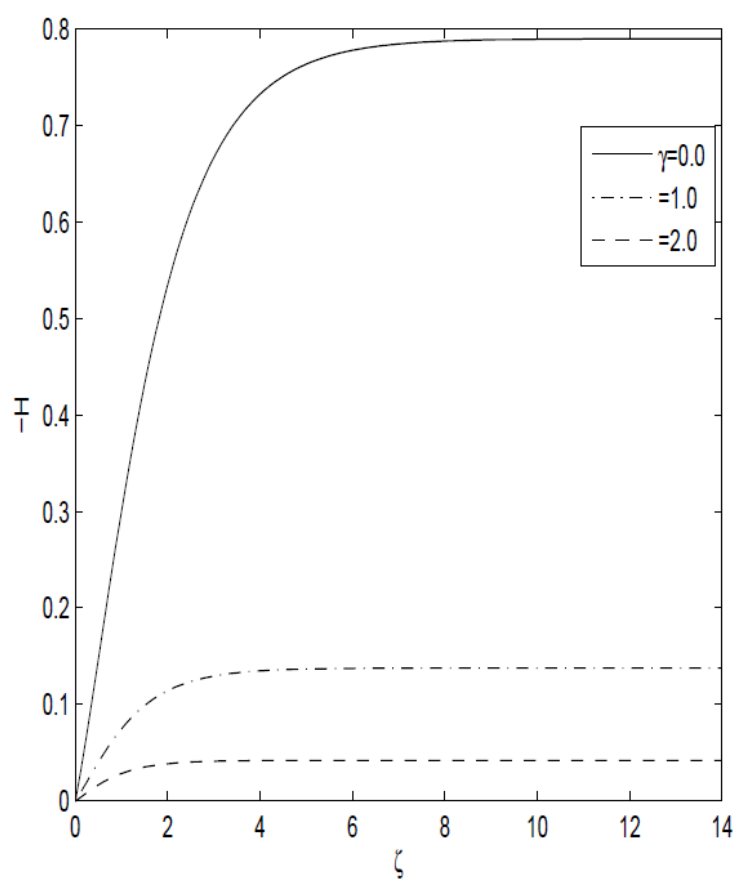

(b)

Fig. 4. Axial distributions of the normalized axial velocity component $-H$ with (a) the velocity slip parameter $\lambda(=\eta)$ for $\gamma=1$ and (b) the porosity parameter $\gamma$ for $\lambda=\eta=1$.

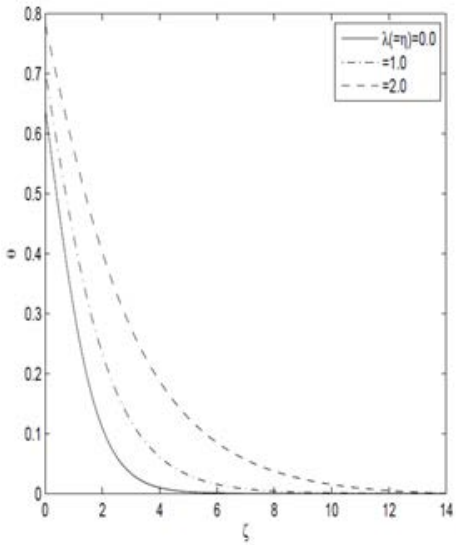

(a)

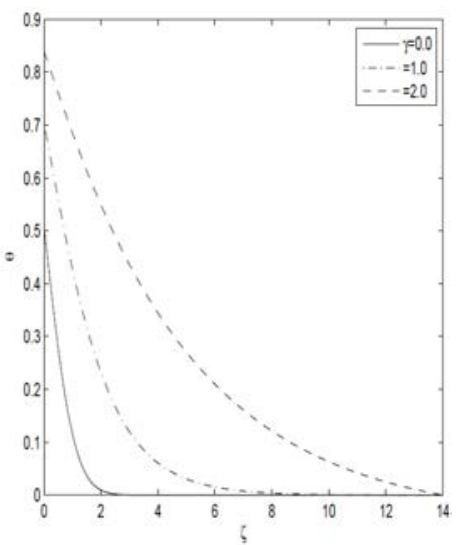

(b)

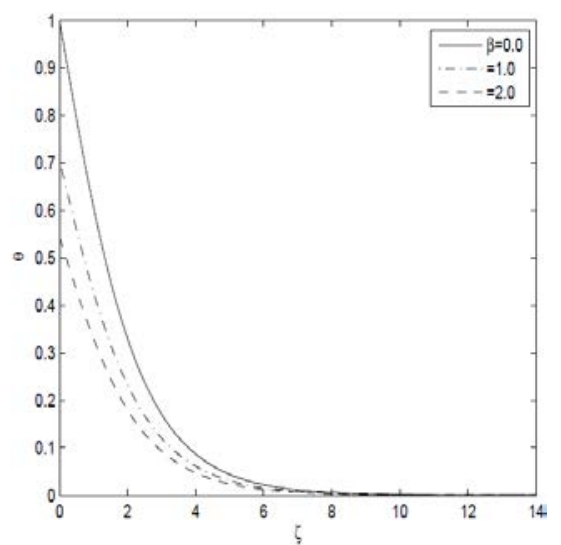

(c)

Fig. 5. Axial distributions of the normalized temperature $\theta$ for various values of (a) the velocity slip parameter $\lambda(=\eta)$ for $\gamma=\beta=1$, (b) the porosity parameter $\gamma$ for $\lambda=\eta=\beta=1$ and (c) the thermal slip parameter $\beta$ for $\gamma=\lambda=\eta=1$.

One interesting quantity for practical applications is the power required to overcome the frictional drag on the disk surface or to maintain the disk at a constant rotation rate $\Omega$. The dimensionless moment coefficient is then defined as: $C_{m}=-\pi G^{\prime}(0) / R e$, where $R e=\Omega R^{2} / v$ is the Reynolds number fixed here to $R e=0.01$. Note that $R e=0.01$ has been chosen arbitrarily for more convenience but it remains within the validity range of the equation system (6)-(9). This definition of $C_{m}$ is the extension to the finite disk problem, which 


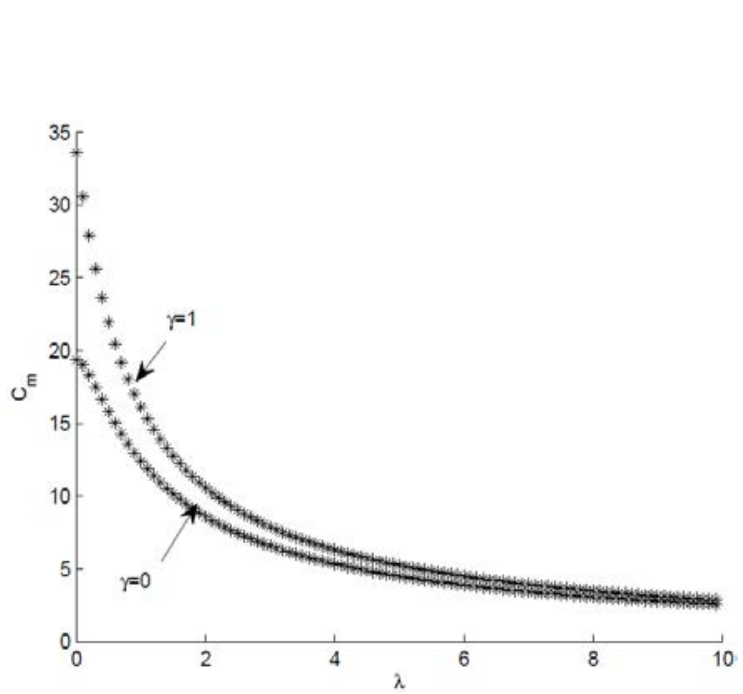

(a)

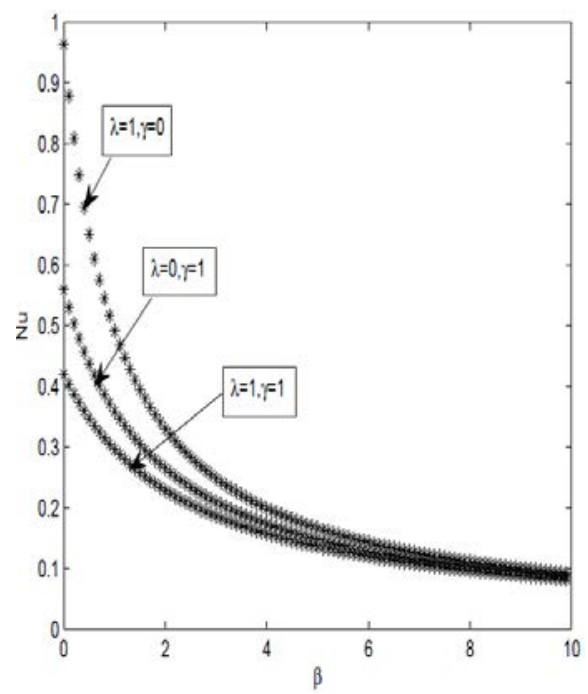

(b)

Fig. 6. (a) Variations of the moment coefficient $C_{m}$ with the velocity slip parameter $\lambda(=\eta)$ for $\gamma=0$ and $\gamma=1$; (b) Variations of the Nusselt number $N u$ with the thermal slip parameter $\beta$ when $(\gamma=0, \lambda=1),(\gamma=1, \lambda=0)$ and $(\gamma=\lambda=1)$.

supposes that the disk radius is large enough. Its variations with $\lambda$ are plotted in Fig. 6(a) for two values of the porosity parameter $\gamma=0$ and $\gamma=1$. It is to be compared to the value obtained $C_{m}=-242.8$ by Sahoo et al. [14] for a viscous Bödewadt flow with $\gamma=\lambda=0$. In the present case, the axial gradient of $G$ at $\zeta=0$ is indeed here negative, such that the moment coefficient is always positive. As expected, $C_{m}$ decreases exponentially with $\lambda$ whatever the value of $\gamma$. For a porous disk with a porosity parameter equal to $\gamma=1$, the moment coefficient is increased significantly for small values of the slip parameter. On the contrary, for large values of $\lambda$ (around 10 ), the porosity parameter has only a very weak influence.

Finally, the variations of the Nusselt number are exhibited in Fig. 6(b). It is noteworthy that for $\beta=\lambda=0$ (no slip) and $\gamma=0$ (impermeable disk), $N u$ is equal to 1 for laminar flows [5]. Thus, when partial slip is imposed at the surface of a non-porous rotating disk $(\lambda=1$ and $\gamma=0)$, the Nusselt number is slightly reduced compared to the basic case previously evoked with $N u=0.97$ for $\beta=0$. It shows in particular that, as expected, the mean flow field has only a weak effect on the thermal one. On the contrary, the porosity parameter has a prominent effect on the heat transfer coefficient. As example, for a porous disk with no-slip conditions $(\gamma=1, \lambda=0), N u$ is reduced by almost a factor 2 for $\beta=0$. Whatever the values of $\gamma$ and $\lambda$ considered here, $N u$ is found to decay exponentially in a monotonic fashion as the thermal slip factor is increased to tend to an asymptotical value slightly lower than 0.1 for $\beta=10$.

\section{CONCLUSION}

In this note, the steady von Kármán flow and the heat transfer in a porous medium have been numerically studied. A direct multiple shooting method has been adopted to solve the resulting coupled, highly nonlinear system of differential equations. The results show the various effects of the porosity and slip parameters on the velocity and temperature profiles. Increasing the porosity parameter $\gamma$ induces a decrease of all velocity components and an increase of the temperature throughout the domain of integration. It has besides a dominating influence on the heat transfer coefficient compared to the velocity slip parameter $\lambda$. The moment coefficient to maintain the disk at a constant rotation rate is also enhanced by increasing values of $\gamma$. The 
temperature jump or an increase of the temperature slip factor $\beta$ acts in favor of a decrease of the Nusselt number, which tends to 0.1 for $\beta \simeq 10$.

\section{REFERENCES}

1. Bonnecaze, R., Mano, N., Nam, B. and Heller, A., "On the behavior of the porous rotating disk electrode", Journal of the Electrical Society, Vol. 154, No. 2, pp. F44-F47, 2007.

2. Hwang, K. and Lin, S., "Filtration flux-shear stress-cake mass relationships in microalgae rotating-disk dynamic microfiltration", Chemical Engineering Science, Vol. 244, pp. 429-437, 2014.

3. von Kármán, T., "Über laminare und turbulente Reibung", ZAMM - Journal of Applied Mathematics and Mechanics, Vol. 1, pp. 233-252, 1921.

4. Rott, N. and Lewellen, W., Boundary Layers in Rotating Flows, Applied Mechanics, Springer Verlag, 1966.

5. Owen, J. and Rogers, R., Flow and Heat Transfer in Rotating-Disc Systems, Vol. 1: Rotor-Stator Systems, John Wiley \& Sons, 2009.

6. Zandbergen, P. and Dijkstra, D., "Von Kármán swirling flows", Annual Review of Fluid Mechanics, Vol. 19, pp. 465-491, 1987.

7. Miklavčič, M. and Wang, C., "The flow due to a rough rotating disk", The Journal of Applied Mathematics and Physics (ZAMP), Vol. 55, pp. 235-246, 2004.

8. Turkyilmazoglu, M. and Senel, P., "Heat and mass transfer of the flow due to a rotating rough and porous disk", International Journal of Thermal Sciences, Vol. 63, pp. 146-158, 2013.

9. Joseph, D., "Note on steady flow induced by rotation of a naturally permeable disk", Quarterly Journal of Mechanics and Applied Mathematics, Vol. 18, No. 3, pp. 325-331, 1965.

10. Attia, H., "Steady flow over a rotating disk in porous medium with heat transfer", Nonlinear Analysis: Modelling and Control, Vol. 14, pp. 21-26, 2009.

11. Attia, H., "Asymptotic solution for rotating disk flow in a porous medium", Mechanics and Mechanical Engineering, Vol. 14, No. 1, pp. 119-136, 2010.

12. Rashidi, M., Mohimanian Pour, S., Hayat, T. and Obaidat, S., "Analytic approximate solutions for steady flow over a rotating disk in porous medium with heat transfer by homotopy analysis method", Computers and Fluids, Vol. 54, pp. 1-9, 2012.

13. Whitaker, S., "The Forchheimer equation: A theoretical development", Transport in Porous Media, Vol. 25, pp. 27-61, 1996.

14. Sahoo, B., Abbasbandy, S. and Poncet, S., "A brief note on the computation of Bödewadt flow with Navier slip boundary conditions", Computers and Fluids, Vol. 90, pp. 133-137, 2014.

15. Wang, C., "Stagnation slip flow and heat transfer on a moving plate", Chemical Engineering Science, Vol. 61, pp. 7668-7672, 2006.

16. Broyden, C., "On the discovery of the 'good Broyden method", Mathematical Programming, Series B, Vol. 87, pp. 209-213, 2000.

17. Sahoo, B., "Effects of partial slip, viscous dissipation and Joule heating on Von Kármán flow and heat transfer of an electrically conductive non-Newtonian fluid", Communications in Nonlinear Science and Numerical Simulation, Vol. 14, pp. 2982-2998, 2009. 